\title{
Klinisch relevante Erkenntnisse für „eilige Leser“
}

Auch die DGIM Gesellschaftsaugabe 4/2011 der Deutschen Medizinischen Wochenschrift verspricht dem „eiligen“ Leser in Kürze klinisch relevante Ergebnisse aus den 11 Schwerpunkten der Inneren Medizin zu vermitteln. Die Autoren wählen zu einem Themenschwerpunkt ihres Gebietes die in der Regel „hochrangig“ publizierten Erkenntnisse der letzten Jahre aus. Ich möchte allen Autoren danken, dass sie zeitgerecht die Literatur gesichtet haben und die Daten leserfreundlich, mit eigenen Interpretationen versehen, präsentieren. Etliche Ergebnisse werden mit hoher Wahrscheinlichkeit Eingang in unser klinisches Handeln finden. Erlauben Sie mir, auf einige dieser „Neuigkeiten“ stichpunktartig einzugehen.

Angiologie: Die Geschichte der Medizin zeigt uns immer wieder, dass auch „logisch“ erscheinende medizinische Maßnahmen, werden sie wissenschaftlich im Rahmen von prospektiven randomisierten Studien evaluiert, oft als Mythen entlarvt werden. So muss nach einer neuen S3-Leitlinie zur Thromboembolieprophylaxe der Einsatz von Stützstrümpfen völlig neu überdacht werden. Nur noch bei viszeralchirurgischen Eingriffen mit hohem Thromboembolie-Risiko zeigte eine Studie eine Wirksamkeit.

Endokrinologie und Diabetologie: Eine pharmakologische Therapie der Adipositas, u.a „Appetitzügler" ist vorerst noch nicht in Sicht. Außer der medikamentösen Hemmung der Lipase mit der in Pilzen entdeckten Substanz Tetrahydrolipstatin (Orlistat) mussten andere Medikamente aufgrund von Nebenwirkungen, wie Depression oder erhöhtes kardiovaskuläres Risiko, vom Markt genommen werden; andere Wirkprinzipien wurden gar nicht erst zugelassen. Letztlich ist unser Verständnis der hormonellen und nervalen Regulation des Sättigungs- und Hungergefühls noch unvollständig, um eine gezielte und nebenwirkungsarme pharmakologische Beeinflussung dieser Regelkreise zu erreichen. Genetisch sind wir seit Jahrtausenden auf den Umgang mit Nahrungsmangel programmiert. Jetzt wird in den Industrienationen Nahrung an sprichwörtlich jeder Ecke angeboten.

Geriatrie: Harninkontinenz ist ein häufiges Symptom, das vor allem ältere, oft multimorbide Frauen betrifft und von vielen Patienten aus Schamgefühl verschwiegen wird. Die medikamentös-therapeutischen Möglichkeiten sind noch nicht zufriedenstellend. In dem Beitrag wird die Notwendigkeit eines geriatrischen „Ba-
sis-Assessments" dargestellt, welches über das weitere Procedere entscheidet. Es gilt die verschiedenen Formen der Inkontinenz und Mischformen zu kennen. Die meisten geriatrischen Patienten erhalten ja aufgrund der Multimorbidität eine Vielzahl an Medikamenten. Je nach Inkontinenzart müssen die Medikamente bekannt sein, die die Inkontinenz verschlechtern. Die in diesem Artikel enthaltene Tabelle 1 ist besonders aussagekräftig und handlungsrelevant.

Hämatologie und Onkologie: In keinem anderen Schwerpunkt der Inneren Medizin sind die Erfolge der "targeted therapy“ so eindrucksvoll wie in der Hämatologie und Onkologie. So kann bei einer früher tödlichen Erkrankung wie der chronisch-myeloischen Leukämie mit Tyrosinkinase-Inhibitoren, wie Imatinib, durch Hemmung von Signal-Transduktionskaskaden die Proliferation der Leukämiezellen gehemmt werden. Der Beitrag „von der risikoadaptierten Therapie zur Genotyp-spezifischen Therapie“ zeigt weitere beeindruckende Beispiele auch bei der akuten myeloischen Leukämie. Hier lassen sich durch genotypische Charakterisierung der Leukämie, z.B. Nachweis bestimmter Mutationen, Risikogruppen identifizieren, die das weitere therapeutische Procedere bestimmen, wie Art der Chemotherapie, Allokation zur Stammzelltransplantation, Konsolidierungstherapie.

Infektiologie: Bei der HIV-Infektion ist Dank der therapeutischen Möglichkeiten das Risiko der Malignomentwicklung zurückgegangen, aber nicht für alle Tumoren. Die Ursache der Zunahme des Zervixkarzinoms bedarf der Klärung. Diskutiert wird, wann und für welche Malignome, z.B. Lymphom, hepatozelluläres Karzinom und Analkarzinom, regelmäßige Vorsorgeuntersuchungen den Patienten angeboten werden sollten.

Intensivmedizin: Zuviel, aber auch zu wenig Sauerstoff nach Wiederherstellung des Kreislaufs erhöhen neurologische Defizite in der Spätfolge; Hypothermie nach überlebtem plötzlichen Herztod hingegen ist neuroprotektiv.

Kardiologie: Sind beim Vorhofflimmern die Tage der Antikoagulation mit Pheprocoumon gezählt, da andere Medikamente, die die Gerinnung beeinflussen, die gleiche Wirksamkeit zur Embolieprophylaxe, aber ein geringeres zerebrales Blutungsrisiko haben? Hier sei der Thrombinantagonist Dabigatran genannt. Die Therapie des

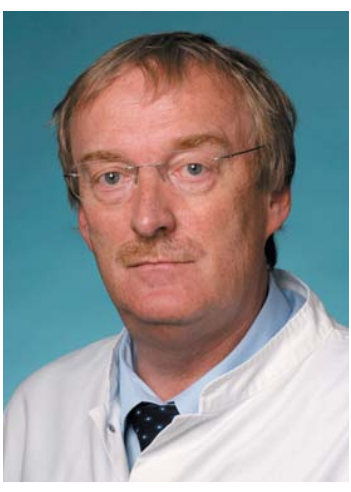

J. Mössner

Institut

Klinik und Poliklinik für Gastroenterologie und Rheumatologie,

Department für Innere

Medizin, Neurologie und Dermatologie,

Universitätsklinikum Leipzig

Bibliografie

DOI $10.1055 / \mathrm{s}-0031-1281552$

Dtsch Med Wochenschr 2011;

136: 1565-1566 - (c) Georg

Thieme Verlag KG Stuttgart . New York · ISSN 0012-0472

Korrespondenz

Prof. Dr. med. Joachim Mössner Klinik und Poliklinik für Gastroenterologie und Rheumatologie Department für Innere Medizin, Neurologie und Dermatologie Universitätsklinikum Leipzig AöR

Liebigstr. 20

04103 Leipzig

Tel. 0341/97-12200

Fax 0341/97-2209 
Vorhofflimmerns und Embolieprophylaxe ist jedoch noch wesentlich differenzierter, um nur Katheterablation und perkutaner Verschluss des linken Vorhofohres zu nennen.

Nephrologie: Bei der chronischen Niereninsuffizienz ist die Messung von Progressionsfaktoren zur Prognoseabschätzung und Therapiesteuerung wichtig. Die therapeutische Hemmung des Renin-Angiotensin-Systems bleibt unverzichtbar. Das chronische Nierenversagen wird aufgrund der Zunahme des Typ-2-Diabetes und der Hypertonie zu einem der wichtigsten Gesundheitsprobleme werden. Komorbidäten, wie Herzinfarkt, sind häufig Prognose-entscheidend. „Erfreulicherweise" wird weniger als $1 \%$ der Patienten mit chronischer Niereninsuffizienz dialysepflichtig.

In der Pneumologie bieten sich überzeugende diagnostische und therapeutische Möglichkeiten der Endoskopie an, zum Beispiel die Endosonographie-gesteuerte transbronchiale Biopsie, die die Treffsicherheit erhöht oder die endoskopische Volumenreduktion in der Behandlung des Emphysems.

Rheumatologie: Gastrointestinale Nebenwirkungen der NSAR bis hin zu lebensbedrohlichen Ulkusblutungen sind bekannt. Wie bereits vermutet, haben auch die „klassischen“ NSAR ein erhöhtes kardiovaskuläres Risiko. Ihre frühere Zulassung unterlag sicher nicht den strengen Kriterien, wie sie bei der Zulassung heute verlangt werden. Das erhöhte kardiovaskuläre Risiko führte ja zum „Aus“ u.a. für das bezüglich des Gastrointestinaltraktes im Vergleich zu nicht selektiven NSAR verträglichere Coxib Rofecoxib. Wir dürfen daher gespannt den Einsatz möglicher medikamentöser Alternativen zur Schmerztherapie, wie des Opioids Tapentadol, erwarten.

Verdauungs- und Stoffwechselkrankheiten: Hier werden neue Ansätze zur Behandlung der Komplikationen der Leberzirrhose besprochen. Bei Leberzirrhose korreliert ein portalvenöser Druck $>20 \mathrm{~mm} \mathrm{Hg}$ mit einem erhöhten Ösophagusvarizen-Blutungsrisiko, insbesondere einem erhöhten Rezidivblutungsrisiko. In dieser Situation ist der transjuguläre intrahepatische portosystemische Stent-Shunt wahrscheinlich die Therapie der Wahl im Vergleich zur Ligaturbehandlung. Die therapeutischen Optionen zur Behandlung der hepatischen Enzephalopathie beschränkten sich bislang auf Laktulose und verzweigtkettige Aminosäuren. Nach dem Ergebnis aktueller Studien sollte das nicht resorbierbare Antibiotikum Rifaximin für diese Indikation zugelassen werden.
Ich empfehle den Leserinnen und Lesern aber auch die weiteren Beiträge dieses Heftes, so die differenzialdiagnostischen Überlegungen, die zur Lösung des Mediquiz erforderlich sind. Die Einnahmetreue von Medikamenten („adherence“) ist nicht nur bei multimorbiden Patienten ein großes Problem und kann die Therapie der Erkrankung konterkarieren. Der Artikel von Laufs et al. gibt hier gute Tipps. Die Prävention der Adipositas bei Kindern ist eine der wichtigsten Herausforderungen, um der Epidemie der Adipositas mit all ihren negativen Folgen entgegenzuwirken. Auf dieses Problem weist der Beitrag von G. Kühne hin. In Leipzig ist unter der Leitung von Professor Dr. med. Michael Stumvoll zum Thema Adipositas ein IFB (integriertes Forschungs- und Behandlungszentrum) entstanden, das auch das Thema Adipositas bei Kindern umfasst. Die Kasuistik von Karch et al. macht auf eine seltene Tumorentität des Ösophagus aufmerksam, die man kennen sollte.

\section{Mit freundlichen kollegialen Grüßen}

Ihr

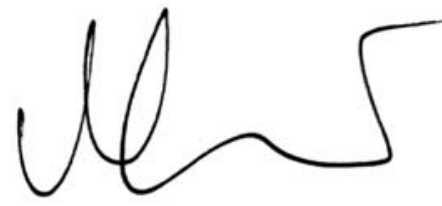

Prof. Dr. med. Joachim Mössner 